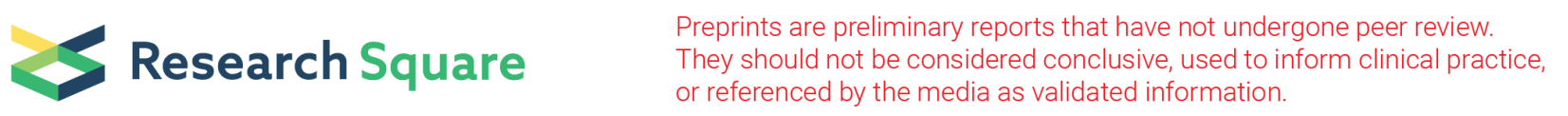

\title{
Multi-scale neural signatures of major depressive, anxiety, and stress-related disorders
}

\section{Peter Zhukovsky}

University of Cambridge https://orcid.org/0000-0002-7181-2095

\section{Michael Wainberg}

Centre for Addiction and Mental Health

\section{Milos Milic}

Centre for Addiction and Mental Health

\section{Shreejoy Tripathy}

Centre for Addiction and Mental Health

\section{Benoit Mulsant}

Centre for Addiction and Mental Health

\section{Daniel Felsky}

Centre for Addiction and Mental Health

\section{Aristotle Voineskos ( $\square$ aristotle.voineskos@camh.ca )}

Campbell Family Mental Health Research Institute Centre for Addiction and Mental Health https://orcid.org/0000-0003-0156-0395

\section{Article}

Keywords: Major Depression Disorder, Anxiety, Stress-related disorders, Cognitive Function, Trailmaking, Processing Speed, Functional Connectivity, Cortical Thickness, Partial Least Squares, UK Biobank

Posted Date: July 27th, 2021

DOI: https://doi.org/10.21203/rs.3.rs-711822/v1

License: (ㅇ) (i) This work is licensed under a Creative Commons Attribution 4.0 International License. Read Full License 


\section{Abstract}

The extent of shared and distinct neural mechanisms underlying major depressive disorder (MDD), anxiety and stress-related disorders is still unclear. We compared the neural signatures of these disorders in 5,405 UK Biobank patients and 21,727 healthy controls. We found the greatest case-control differences in resting-state functional connectivity and cortical thickness in MDD, followed by anxiety and stress-related disorders. Neural signatures for MDD and anxiety disorders were highly concordant, namely in altered frontostriatal connectivity, whereas stress-related disorders showed a distinct pattern. Controlling for cross-disorder genetic risk somewhat increased the similarity between functional neural signatures of stress-related disorders and both MDD and anxiety disorders. Among cases and healthy controls, reduced within-network and increased between-network frontoparietal and default mode connectivity was associated with poorer cognitive performance (processing speed, attention, associative learning and fluid intelligence). These results provide evidence for distinct neural circuit function impairments in MDD and anxiety disorders compared to stress disorders; yet cognitive impairment appears unrelated to diagnosis and varies with circuit function.

\section{Introduction}

Major depressive disorder (MDD) and anxiety disorders (i.e., generalized anxiety disorder, panic disorder without agoraphobia) are highly comorbid psychiatric disorders ${ }^{1-3}$, with shared epidemiologic and developmental features ${ }^{4}$ and a shared genetic basis, ${ }^{5}$ and are among the leading causes of disability worldwide ${ }^{6}$. Depression and anxiety are often triggered by stressful life events, thus sharing the aetiology of stress-related disorders that are defined by occurrence of a severe stressor or trauma (DSM 5). More specifically, posttraumatic stress disorder (PTSD) is characterized by hyperarousal states during recurring flashbacks to the stressful event, while stress adjustment disorder is characterized by depressive symptoms in response to a severe stressor ${ }^{7}$. Unlike MDD and anxiety disorders, which are recurrent or chronic, stress adjustment disorder resolves within 6 months after termination of the stressor. While considerable neurobiological research has been conducted at a disorder-specific level, few studies have investigated a broad spectrum of MDD, anxiety, and stress disorders to examine shared and distinct neural correlates.

Task-based functional MRI findings point to disrupted emotional processing and executive dysfunction, exemplified by disrupted cognitive control ${ }^{89}$, across a variety of disorders, including, but not limited to MDD and anxiety disorders. Similarly, gray matter reductions have been shown in the insular and anterior cingulate cortices across mood, anxiety, and psychotic disorders ${ }^{10,11}$. Further, greater similarity of deviations in brain structure was found for disorders with greater genetic similarity ${ }^{12}$. Such transdiagnostic phenotypes aim to capture the shared neurocognitive basis of symptoms presenting across disorders and could have utility in improving psychiatric nosology ${ }^{13}$.

Inferior prefrontal and insular cortex, the inferior parietal lobule and the putamen are hypoactivated in task-based functional magnetic resonance imaging (fMRI) paradigms across MDD, anxiety disorders, and stress-related disorders ${ }^{14}$, implicating inhibitory control and salience processing as shared neural phenotypes underlying mood, anxiety, and stress-related disorders. Impairments in executive functions such as inhibitory control over emotional reactivity and negative mood may capture a transdiagnostic dimension of psychopathology ${ }^{15,16}$. Executive function is also impaired by anxiety, which reduces cognitive flexibility and working memory ${ }^{17}$ and impairs attentional control ${ }^{18}$. While some evidence also points towards executive dysfunction in PTSD ${ }^{19,20}$, psychological theories of posttraumatic stress typically emphasize the effects of the traumatic event on memory ${ }^{21,22}$. Executive dysfunction may be linked to both dysregulated mood in MDD and heightened emotional reactivity in anxiety disorders ${ }^{23}$, and thus provides a promising transdiagnostic treatment target.

Here, we leveraged multimodal data from the UK Biobank to determine unique and shared features of brain structure and function in MDD, anxiety disorders, and stress-related disorders, as well as the relationship of such neuroimaging measures to several aspects of cognitive function across these disorders. The UK Biobank includes mid-life and older adults and is thus suitable for investigating cognition related to healthy and abnormal ageing. We selected Trailmaking performance ${ }^{24}$, digit-symbol substitution ${ }^{25}$, fluid intelligence ${ }^{26}$, and paired associate learning ${ }^{27}$ to measure key domains of cognitive function. We used second-order statistical comparisons to investigate genetic and environmental contributions to disorder similarity. We expected to find default mode and frontostriatal connectivity differences in MDD ${ }^{28}$ and anxiety disorders ${ }^{29,30}$, resulting in shared neural signatures. We also hypothesized reduced cortical thickness of the frontoparietal regions in both MDD and anxiety disorders ${ }^{31-33}$, and a separate neural signature of stress-related disorders focused on the hippocampal regions ${ }^{34}$. We hypothesized that co-varying for heritable risk would alter the correlation of crossdisorder neural signatures most highly for the stress-related disorders, given that these disorders are under stronger environmental 
influence and do not show genetic correlations with other mental disorders ${ }^{5}$. Finally, we expected connectivity of frontoparietal, attention, and default mode networks to underlie cognitive performance across disorders.

\section{Methods}

\section{Data}

Healthy controls. Participants were recruited through the UK Biobank (UKB). The overall number of people with UKB preprocessed MRI outputs was 40,669 . Healthy controls were defined by excluding participants who had any one of over 142 ICD-10 codes related to conditions that could affect neural connectivity and structure such as epilepsy and Alzheimer's disease, thus resulting in 21,727 healthy controls (Supplementary Information).

Patients. We used linked health record data available from inpatient and primary care information. ICD-10 codes F32 (a major depressive episode; UKB Data-Field 130895), F33 (recurrent major depressive episodes, UKB Data-Field 130897), F41 (generalized anxiety disorder, panic disorder without agoraphobia, other mixed, specified, and unspecified non-phobic anxiety disorders; Data-Field 130907), and F43 (reaction to severe stress such as post-traumatic stress disorder, and stress adjustment disorders; UKB Data-Field 130911) were used to define four mutually exclusive patient groups. These groups included participants with lifetime diagnoses of 1) MDD but not anxiety or stress-related disorders ("MDD-"), 2) non-phobic anxiety disorders but not MDD or stress-related disorders ("ANX-"), 3) comorbid MDD and anxiety but not stress-related disorders ("MDD + ANX"), and finally 4) stressor related disorders but not MDD or anxiety ("STR-"). Case definition was based on lifetime diagnoses as we wanted to investigate the neural and genetic signatures of vulnerability for and consequences of the psychiatric disorders.

Depression symptoms at the time of MRI scan were assessed using a cut-off score of 2 on the PHQ-2 ${ }^{35}$ (UKB Data-Fields 2050 and 2060); self-reported restlessness and tiredness indices were also included (Data-Fields 2070 and 2080, respectively).

Neuroimaging data acquisition. Briefly, MRI data were collected on Siemens 3T Skyra and 32-channel receive head coil (T1-weighted structural sequence $T R=2,000 \mathrm{~ms}, T E=2.01 \mathrm{~ms}, \mathrm{TI}=880 \mathrm{~ms}$, flip angle $=8^{\circ}$, resolution $=1 \mathrm{~mm} 3$ ). Multiband gradient echo echo planar imaging (EPI) sequence (length $=6 \mathrm{~min}$, field of view $[\mathrm{FOV}]=210 \mathrm{~mm}$, slices $=64, \mathrm{TR}=735 \mathrm{~ms}, \mathrm{TE}=39 \mathrm{~ms}$, resolution $=2.4 \mathrm{~mm} 3$ ) was used to acquire resting-state functional MRI scans. More detail on the imaging protocols can be found in ${ }^{36}$. Structural images were processed using Freesurfer ${ }^{37,38}$, while functional images were processed using FMRIB's MELODIC and FSLnets tools ${ }^{36,39}$. If images were flagged as "unusable" by UKB's automated quality control pipeline, they were excluded from analysis.

Neuroimaging processing. Two neuroimaging modalities were included: resting-state functional connectivity and cortical thickness. We used 210 resting-state connectivity features comprising all pairs of partial correlations among the 21 independent components (ICs) identified by the UKB preprocessing pipeline in an independent component analysis (Data-Field 25752). We mapped 19 of the 21 independent components that were primarily located in the neocortex to Yeo7 networks ${ }^{40}$ by comparing the proportions of voxels in each component (thresholded at $z>3$ ) that fell into each of the 7 networks. One component primarily encompassed the cerebellum, while another component encompassed subcortical regions (notably the striatum).

We also used UKB-provided Freesurfer outputs (Data-Field 20227) to derive cortical thickness values for the 360 regions in the HCP parcellation ${ }^{41}$. HCP labels were first registered from fsaverage space to subject space (mri_label2label) and summary statistics for each label were generated (mri_segstats). These derivatives will be made available through the UKB Returns Catalogue (https://biobank.ctsu.ox.ac.uk/crystal/docs.cgi?id=1, Project ID: 61530).

Cognitive data. We used four cognitive tests assessing executive function, processing speed, and learning: time to complete the alphanumeric path of the trail-making test (TMT, Data-Fields 6350 and 6351) as a proxy for visuospatial processing speed and executive function ${ }^{42}$, fluid intelligence (Data-Field 20016), paired-associates learning (PAL, Data-Field 20197) as a measure of memory and associative learning ${ }^{43}$, and the digit-symbol substitution task (DSST, Data-Field 23324) as a measure of attention, visuoperceptual speed and associative learning ${ }^{44}$. In addition to testing cognitive function, TMT and DSST also tap into participants' motor speed as participants with worse ability to write and draw will be at a disadvantage. Cognitive test data were collected during the same visit as the MRI scan. More information can be found in the Supplement.

Genetic data. A polygenic risk score (PRS) for MDD was derived from public genome-wide association study (GWAS) results ("PGC MDD No UKB / No 23andMe" at https://www.med.unc.edu/pgc/download-results/) (Wray et al., 2018) as described previously ${ }^{45}$. The 
summary statistics for ANX were taken from the Integrative Psychiatric Research (iPSYCH) consortium ${ }^{46}$, at https://ipsych.dk/fileadmin/ipsych.dk/Downloads/daner_woautism_ad_sd8-sd6_woautismad_cleaned.gz. These results were derived from a GWAS with 4,584 patients with anxiety disorders and 19,225 controls with no anxiety, stress-related or mood diagnoses. The PTSD summary statistics were obtained from the Psychiatric Genetics consortium (PGC) at https://www.med.unc.edu/pgc/downloadresults $^{47}$. Briefly, the UKB's imputed genotypes were filtered to autosomal, non-duplicate single-nucleotide variants with imputation INFO score $>0.8$, and with Hardy-Weinberg equilibrium p-value $>10^{-10}$, missingness $<5 \%$ and minor allele frequency $>0.1 \%$ across selfreported white participants. Summary statistics were harmonized with the UKB with respect to reference/alternate allele and strand, then subset to $p<0.05$ and pruned to $r^{2}<0.2$ using frequency-informed linkage disequilibrium pruning. Before computing associations between the MDD PRS and MRI-derived features, the first 10 principal components of genetic variance (available from https://pan.ukbb.broadinstitute.org/downloads) were regressed out of the PRS.

\section{Statistical analysis}

Effect of diagnosis and polygenic risk on neuroimaging and cognition. We tested for case-control differences in partial correlations between functional ICA components as well as regional cortical thickness using separate general linear models (fit/m, anova, MATLAB R2016a). Sex, age, age ${ }^{2}$, age $\times$ sex, average head motion during the resting-state fMRI run, and UKB imaging acquisition site were included as covariates. These models were specified as follows:

Pairwise connectivity $\sim$ group $_{D x}+$ age + age $e^{2}+$ age $\times \operatorname{sex}+\operatorname{sex}+$ head motion + site

Regional cortical thickness $\sim$ group $_{D x}+a g e+a g e^{2}+a g e \times s e x+s e x+$ site

We re-analyzed the data using linear models including PRS for MDD, anxiety disorders, and PTSD as covariates. This analysis allowed us to estimate the effect of heritable MDD, ANX and PTSD risk on functional connectivity and cortical thickness measures. These models were specified as follows:

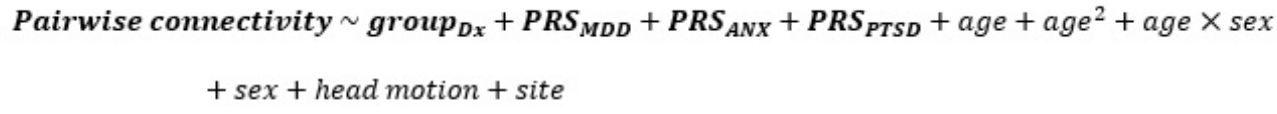

Permutation testing ( $\left.n=1,000, P_{\text {PERM }}<0.05\right)$ was used to test whether diagnostic group and PRS had a significant effect on functional connectivity and cortical thickness. When a significant group effect (omnibus F-test) was present, each of the case groups was compared to the healthy control group. We used Bonferroni correction on the post hoc tests $(p<0.0125)$ to assess significance.

Further, we used linear models to test for an effect of group and PRS on the cognitive function variables while covarying for sex, age, age $^{2}$, age $\times$ sex, and testing center. For greater interpretability, we converted the resulting t-statistics to standardized effect sizes (Cohen's ds) via the following transformation 48 :

$$
d=\frac{2 * \mathrm{t}}{\sqrt{d f}}
$$

Disorder similarity. For each case-control comparison (i.e., MDD- vs control, ANX- vs control, MDD + ANX vs control, and STR- vs control), the above-mentioned linear models generated $210+360$ t-statistics, one for each functional connectivity and cortical thickness feature. To assess the degree of cross-disorder concordance in MRI signatures, we computed Pearson's correlations between each pair of disorders across the 210 functional connectivity t-statistics and, separately, across the 360 regional cortical thickness t-statistics. The statistical significance of these correlations was assessed via permutation testing: random distributions of case-control t-statistics were generated by re-running the linear models with permuted outcome variables and generating a random distribution of correlations between these t-statistics (all code available at https://www.github.com/peterzhukovsky). 
Partial correlation analyses covarying for MDD PRS were used to assess the degree to which MDD PRS was contributing to pairwise disorder similarity.

Neural correlates of cognitive function in anxiety and MDD. Partial least squares (PLS) regression was used to assess the relationship between functional connectivity and cognitive function at the time of the imaging visit. A separate PLS regression was used to test for multivariate associations between regional cortical thickness and cognitive performance. Model significance was tested using permutation testing following previous studies ${ }^{49}$. In these analyses, we focused on participants in all case groups with no missing cognitive data $(n=3,216)$. We regressed out age, sex and site from both neuroimaging and cognitive variables; we also regressed out average head motion from the $\mathrm{fMRI}$ data. We used $\mathrm{z}$-scored residuals from these regressions to form the predictor matrix $\mathrm{X}(3,216 \times 210)$ and the outcome matrix $Y(3,216 \times 4)$. We repeated the PLS regression exploring brain-cognition associations in healthy controls $(n=$ $14,199)$ and in each of the four case groups separately (Supplementary Information).

PLS returns a set of components that attempt to maximize the covariance between the PLS scores summarizing $X$ and $Y$. PLS scores are a linear combination of the predictor variables $(X)$ and component loadings. We used bootstrapping $(n=5,000)$ to identify which predictors showed robust contributions to the PLS latent variable. A threshold of $|Z|>3$ was chosen to identify the most robust connectivities associated with cognitive performance ${ }^{49}$.

\section{Results}

Demographic and clinical information for all included UKB participants is summarized in Table 1. 
Table 1

Demographic and clinical sample characteristics. Lifetime diagnosis of MDD (F32/F33), anxiety (F41), stress-related disorders (F43) was used to define the groups. Mean age and mean polygenic risk scores (PRS) ( \pm Standard deviation) are shown. Group effects were assessed using a one-way analysis of variance (F-test) for age and PRS. Chi-square goodness-of-fit tests for categorical comparisons.

The two-item Patient Health Questionnaire (PHQ-2) with a cutoff score of two or greater was used to test for presence of depressed mood in participants at the time of scanning and cognitive testing. This threshold has high PHQ-2 sensitivity (0.91) and specificity (0.67)

for diagnosis made using a semi-structured interview ${ }^{35}$. We show the total numbers of participants with lifetime use of medication

falling into five categories: SSRI, selective serotonin reuptake inhibitor; SARI: Serotonin antagonist and reuptake inhibitors; SNRI, selective noradrenaline reuptake inhibitor; TCA: tricyclic antidepressants; MAO-I, monoamine oxidase inhibitors; NaSSA - Noradrenergic and specific serotonergic antidepressants. Major depressive disorder (MDD-), non-phobic anxiety disorders (ANX-), comorbid MDD and

anxiety (MDD + ANX) and in stress-related disorders (STR-). More information on the medications in each category can be found in Supplementary Table 4. More details on the sample sizes are available in Figure S7.

\begin{tabular}{|c|c|c|c|c|c|c|c|c|c|c|c|c|}
\hline & \multicolumn{2}{|c|}{ Healthy Controls } & \multicolumn{2}{|l|}{ MDD- } & \multicolumn{2}{|c|}{ ANX- } & \multicolumn{2}{|c|}{ MDD + ANX } & \multicolumn{2}{|l|}{ STR- } & \multirow{2}{*}{$\begin{array}{l}\text { Group } \\
\text { Effect }\end{array}$} & \multirow{2}{*}{$\begin{array}{l}P \text { - } \\
\text { value }\end{array}$} \\
\hline $\mathbf{N}$ & 21,727 & & 3,233 & & 664 & & 676 & & 832 & & & \\
\hline Age at MRI & 63.8 & $(7.5)$ & 62.4 & $(7.5)$ & 63.5 & $(7.2)$ & 62.5 & $(7.7)$ & 62.3 & $(7.1)$ & 34.09 & $\begin{array}{l}2.0 \mathrm{E}- \\
28\end{array}$ \\
\hline Nr Female & 10,894 & $(50.1 \%)$ & 2,064 & $(63.8 \%)$ & 409 & $(61.6 \%)$ & 447 & $(66.1 \%)$ & 520 & $(62.5 \%)$ & 320.0 & $\begin{array}{l}5.2 \mathrm{E}- \\
68\end{array}$ \\
\hline $\mathrm{PHQ}-2>=2$ & 1,705 & $(7.8 \%)$ & 977 & $(30.2 \%)$ & 104 & $(15.7 \%)$ & 231 & $(34.2 \%)$ & 103 & $(12.4 \%)$ & 1751.4 & $\begin{array}{l}< \\
0.0001\end{array}$ \\
\hline $\begin{array}{l}\text { Restlessness } \\
>=2\end{array}$ & 3,275 & $(15.1 \%)$ & 1,182 & $(36.6 \%)$ & 198 & $(29.8 \%)$ & 306 & $(45.3 \%)$ & 195 & $(23.4 \%)$ & 1229.5 & $\begin{array}{l}6.4 \mathrm{E}- \\
265\end{array}$ \\
\hline $\begin{array}{l}\text { Tiredness }>= \\
2\end{array}$ & 8,620 & $(39.7 \%)$ & 2,116 & $(65.5 \%)$ & 354 & $(53.3 \%)$ & 455 & $(67.3 \%)$ & 423 & $(50.8 \%)$ & 956.3 & $\begin{array}{l}1.1 \mathrm{E}- \\
205\end{array}$ \\
\hline MDD PRS & -0.21 & $(7.75)$ & 1.20 & $(7.86)$ & 0.83 & $(7.66)$ & 1.33 & (7.91) & -0.02 & (7.61) & 27.8 & $\begin{array}{l}4.8 \mathrm{E}- \\
23\end{array}$ \\
\hline ANX PRS & 0.17 & $(17.07)$ & 0.96 & $(17.07)$ & 1.06 & $(16.20)$ & 1.80 & $(16.85)$ & 0.04 & $(16.51)$ & 3.0 & $\begin{array}{l}1.8 \mathrm{E}- \\
02\end{array}$ \\
\hline PTSD PRS & -0.43 & $(12.01)$ & 2.73 & $(13.15)$ & 1.91 & $(11.97)$ & 3.10 & $(13.48)$ & 0.80 & $(12.39)$ & 57.8 & $\begin{array}{l}1.2 \mathrm{E}- \\
48\end{array}$ \\
\hline \multicolumn{13}{|l|}{$\begin{array}{l}\text { Medication } \\
(\mathrm{N}) \text { : }\end{array}$} \\
\hline SSRI/SARI & 471 & & 891 & & 228 & & 353 & & 175 & & 5417.1 & $\begin{array}{l}< \\
0.0001\end{array}$ \\
\hline SNRI/NRI & 46 & & 119 & & 22 & & 74 & & 18 & & 1063.9 & $\begin{array}{l}5.1 \mathrm{E}- \\
229\end{array}$ \\
\hline TCA & 792 & & 485 & & 164 & & 194 & & 180 & & 1847.7 & $\begin{array}{l}< \\
0.0001\end{array}$ \\
\hline MAO-I & 0 & & 4 & & 0 & & 2 & & 1 & & 39.8 & $\begin{array}{l}4.8 \mathrm{E}- \\
08\end{array}$ \\
\hline NaSSA & 23 & & 107 & & 24 & & 70 & & 16 & & 1125.3 & $\begin{array}{l}2.4 \mathrm{E}- \\
242\end{array}$ \\
\hline
\end{tabular}

Effects of diagnosis and polygenic risk on neuroimaging and cognitive outcomes. In individual comparisons of each diagnostic group to healthy controls, we identified widespread differences in resting state connectivity (Fig. 1A). The most significant differences were found between the Control and MDD- or MDD + ANX groups, whereas smaller differences were found between the Control and ANX- or STRgroups. Specifically, independent component (IC)-12 (presupplementary and supplementary motor areas) showed decreased connectivity with other motor areas (IC-17, superior temporal gyrus) and increased connectivity with the striatal IC-18 across MDD-, MDD + ANX, and ANX- groups, but not STR-

Compared to healthy controls, cortical thickness was reduced across many prefrontal, parietal and temporal regions in the MDD- and MDD + ANX group (Fig. 1B, Supplementary Table 3). In contrast, only select temporal and parietal regions showed a significant decrease 
in cortical thickness in the ANX-group including middle temporal and supramarginal regions. Significantly reduced cortical thickness was also found in the parahippocampal and ventral medial visual regions of the STR- group when compared to Controls.

Finally, significant effects MDD, ANX, and PTSD PRS were observed for both neuroimaging (Supplementary Fig. 4, Supplementary Table 3) and cognitive outcomes (Table 2). As expected, all three PRS had significant negative effects on fluid intelligence, but only PTSD PRS affected other cognitive domains. Importantly, these patterns of PRS effects on cortical thickness and functional connectivity explainable by SNP-based heritable risk for each disorder and can be used to refine our understanding of trait vs. state-based diagnostic signatures.

Case-control differences in cognitive function are summarized in Table 2 and Supplementary Information. Compared to the Control group, the four diagnostic groups showed significant impairments in all domains of cognitive function, except for visuospatial processing (TMT), which was not significantly impaired in MDD + ANX, and paired associate learning, which was not significantly impaired in MDD- or ANX-. Notably, we found impaired TMT, fluid intelligence, and digit-symbol substitution performance in MDD-, ANXand STR- groups, suggesting a transdiagnostic pattern of impairments among different aspects of cognitive function.

Table 2

Cognitive performance in major depressive disorder (MDD-), non-phobic anxiety disorders (ANX-), comorbid MDD and anxiety (MDD + ANX) and in stress-related disorders (STR-). Effects of polygenic risk scores (PRS) for MDD, PTSD and Anxiety disorders are also shown. TMT - Trailmaking test, visuospatial processing; Gf - fluid intelligence; PAL - paired associate learning; DSST - digit symbol substitution test. Significant effects are shown in bold $\left(P_{\text {UNCORRECTED }}<0.05\right)$.

\begin{tabular}{|lllllllll|}
\hline Cognitive Test & Statistic & MDD- & ANX- & MDD + ANX & STR- & MDD PRS & ANX PRS & PTSD PRS \\
\hline TMT & beta & 12.35 & 42.71 & 13.57 & 27.51 & 0.77 & 0.08 & 0.35 \\
\hline Gf & T-stat & 2.56 & 4.27 & 1.54 & 2.89 & 3.92 & 0.94 & 2.92 \\
\hline \multirow{2}{*}{ PAL } & beta & -0.08 & -0.30 & -0.14 & -0.28 & -0.01 & 0.00 & 0.00 \\
\hline \multirow{2}{*}{ DSST } & T-stat & -2.11 & -3.81 & -1.95 & -3.56 & -3.25 & -1.98 & -2.79 \\
\hline & beta & -0.11 & -0.13 & -0.18 & -0.38 & 0.00 & 0.00 & 0.00 \\
& T-stat & -1.95 & -1.10 & -1.70 & -3.25 & 0.03 & -0.47 & -2.63 \\
\hline & beta & -0.70 & -1.50 & -0.54 & -0.65 & -0.01 & 0.00 & -0.01 \\
\hline
\end{tabular}

Similarity of diagnosis-specific neural signatures. After constructing diagnosis-specific, case-control neural signatures, we evaluated the pairwise similarities in their spatial distributions and component effect sizes. A large degree of overlap was found between MDD-, ANX-, and MDD + ANX groups in both functional connectivity and cortical thickness case-control associations (Fig. 2A). On the other hand, the STR- group showed a different spatial pattern of associations that did not resemble the other disorders ( $\left.P_{\text {PERMUTATION }}>0.01\right)$. An exception was the similarity of stress-related disorders and MDD in their effects on resting-state connectivity, which was small but significant.

To determine the degree to which these similarities were moderated by shared heritable risk for these comorbid disorders, we performed the same analysis including all three PRS as co-variates. After controlling for genetic risk, correlations between diagnostic neural signatures for the MDD-, ANX-, and MDD + ANX groups were slightly reduced for both functional and structural measures (Fig. 2B). However, for the STR- group, correlations of functional connectivity signatures were substantially increased (particularly compared with MDD- and MDD + ANX), revealing shared functional pathways between stress disorders and MDD previously obscured by confounding of dissimilar genetic aetiologies. Signatures of cortical thickness were less affected overall.

Neural correlates of cognitive function in MDD-, $A N X$ - $M D D+A N X$, and STR- Given the substantial, albeit variable, overlap in functional neural signatures between the diagnostic groups, we aimed to uncover those circuits specifically related to worse executive function and verbal memory across all disorders $(n=3,216)$ after regressing out age, sex, site and motion confounds. PLS regression identified three 
latent variables that explained $3.1 \%, 1.5 \%$, and $1.3 \%$ of variance in the four cognitive function tests (TMT, $\mathrm{G}_{\mathrm{F}}, \mathrm{PAL}$ and $\mathrm{DSST}$ ). Permutation testing showed that these latent variables together explained a significant amount of variance in cognitive performance $\left(P_{\text {PERM }}<0.001\right)$.

The first latent variable (PLS1) representing functional connectivity, which was optimally associated with cognitive performance captured the most variance in the outcome variables (Fig. 3A) and was associated with worse performance on all four cognitive tests. We found 11 connectivities with normalized PLS1 weights of $Z>3$ (significant and positive) and eight connectivities with normalized PLS1 weights with Z<-3 (significant and negative) (Fig. 3B, 3C). Functional correlates of worse cognitive performance included connectivities of the frontoparietal, default mode and attention networks. Increased DMN-FPN connectivities were also associated with worse cognitive function (e.g., IC-6 with IC-14 or IC-20 with IC-6). Decreased within DMN (IC-20 with IC-14 or IC-7 with IC-9) and within FPN (IC-5 with IC-21 or IC-5 with IC-16) connectivities were associated with worse cognitive function.

The second latent variable (PLS2) captured some variance in fluid intelligence and paired associate learning (Supplementary Figure S5, S6). We found four connectivities with significant positive loadings on PLS2 $(Z>3)$ and one weight with significant negative loading $(Z<-3)$. Higher connectivity of IC-21 (FPN/DMN) with IC-5 (FPN) and IC-1 (DMN) and higher connectivity of the striatal component (IC-18) with the superior temporal gyrus (IC-17) predicted better higher fluid intelligence and associative learning. Lower connectivity of IC-21 (FPN/DMN) with IC-6 (FPN) predicted lower fluid intelligence and associative learning scores. Although the third latent variable (PLS3) was significant at permutation testing, there were no PLS3 weights $|Z|>3$.

To contrast trans-diagnostic signatures with diagnosis-specific signatures, we repeated the PLS regression in each case group separately. We found significant brain-cognition relationships in MDD- and in ANX-but not in MDD + ANX or STR- (Fig. 3C, Supplementary Information), suggesting that former two groups drive the associations across all cases. We found a distinct pattern of brain-cognition relationships in MDD- and ANX-, While components from the FPN and DMN played a major role in brain-cognition relationships in each case group, different connectivities among different components of these networks were implicated in MDD- and ANX-. Many components that were significant (|Z|>3) in MDD- and ANX-separately were also significant in the PLS regression across all cases.

Neural correlates of cognitive function in the Control group. We repeated the PLS analysis in the Control group. Briefly, the PLS model explaining $3.6 \%$ of variance in the cognitive outcomes $\left(P_{P E R M}<0.001\right)$. PLS1 was also linked to worse general cognition in healthy controls. PLS1 weights included independent components from the DMN, FPN and DA/VA network (Supplementary Fig. 6). PLS2 was also linked to worse cognition, similarly to PLS2 in the case group PLS.

\section{Discussion}

In a multimodal investigation of functional connectivity, brain structure, cognitive function, and genetic risk, we found that signatures of MDD and anxiety disorders were highly concordant and distinct from stress-related disorders. Shared polygenic risk explained a small proportion of the similarity in brain connectivity and structure between MDD and anxiety, but moderated the concordance of neural signatures for stress-related disorders and MDD. We further identified impairments in processing speed, attention, fluid intelligence, and paired associate learning shared across all disorders under investigation. A dimensional analysis focusing on MDD, anxiety disorders, and stress-related disorders identified increased between-network and decreased within-network connectivity of the frontoparietal-default mode networks as a neural correlate of poorer cognitive function.

\section{Case-control differences}

The MDD- and MDD + ANX groups showed cortical thickness differences from the control group that were consistent with previous metaanalyses of brain structure in MDD ${ }^{31,32}$ with notable thinning of the parietal and prefrontal cortex (insula, anterior cingulate, inferior frontal, superior frontal and middle frontal gyri, anterior temporal lobe). Comorbid MDD and anxiety are typically associated with greater symptom severity and more limited level of daily functioning ${ }^{50,51}$. Consistent with the expectation of greater disorder severity in comorbid MDD and anxiety disorders, we found additional thinning in the medial temporal regions (hippocampus, entorhinal), suggesting a more profound impact of the comorbid disorders on brain structure. The ANX-group showed localized patterns of cortical thinning in the middle temporal gyri, which is in line with previous evidence of reduced volumes of these regions in anxiety disorders without comorbid MDD ${ }^{11}$. Unlike studies of active anxiety disorders, we did not find significant differences in the anterior cingulate or insular cortices in our ANX-group. This may be explained by the possible time gap between diagnosis and imaging data, as in UKB lifetime diagnosis are recorded and many participants may not be experiencing clinical symptoms at the time of scanning so their brain 
volumes may have recovered since the time of their formal psychiatric diagnosis. Finally, the STR- group also showed localized cortical thinning in the parahippocampal gyrus, and the entorhinal cortex. Parahippocampal and entorhinal cortices are critical to memory formation and retrieval and have been emphasized in studies of PTSD ${ }^{52,53}$. Further, hippocampus has been shown to regulate stress response by influencing the hypothalamic-pituitary-adrenal function ${ }^{34}$. Therefore, stress-induced medial temporal lobe changes are consistent with existing theories of hippocampal function.

Significant resting-state connectivity deviations in people with MDD and MDD with comorbid anxiety were very similar and encompassed a variety of regions that were part of the default mode, frontoparietal, but also subcortical (striatal), cerebellar, and visual networks. Deviations in connectivity within the motor network and between motor and striatal networks were common across the MDD, anxiety disorders, and MDD comorbid with anxiety groups. Our findings are consistent with previously reported alterations in frontoparietal and default mode connectivity in MDD 28 and in anxiety disorders ${ }^{29,30}$. Connectivity between striatal and cortical regions is known to be impaired in MDD ${ }^{54}$ and was also altered in both our MDD groups. Importantly, unlike cortical thickness, functional connectivity was significantly different between the anxiety disorders and the control group, but not in stress-related disorders, reinforcing the differences in neural signatures of this group.

\section{Similarity of neural disorder signatures}

The strong similarity of the neural signatures of the MDD and anxiety disorders groups supports previous studies of shared neural signatures of mental disorders ${ }^{12}$. Controlling for polygenic risk slightly decreased disorder similarity between MDD and anxiety disorders but increased the similarity between stress-related disorders and MDD or anxiety disorders. Since PRSs are representative of trait-like genetic risk for MDD ${ }^{55}$, we conclude that a small proportion of similarity between MDD and anxiety disorders is explained by genetic risk for these disorders. By contrast, stress-related disorders are transient conditions, less affected by genetic risk than MDD or anxiety disorders. Previous evidence shows little genetic similarity between stress-related disorders and MDD or anxiety disorders 5 . However, these disorders share many symptoms and some common presentations. We aimed to capture "state" similarity rather than "state and trait" similarity by covarying for genetic risk, which may increase the similarity between stress-related disorders and MDD and anxiety disorders.

Several neurocognitive processes may underlie the overlap in neural signatures of MDD and anxiety disorders, including executive functioning ${ }^{16}$. A shared neural mechanism could be found in prefrontal regulation of the default mode and limbic circuits responsible for mood and emotional processing. Stress-related disorders, on the other hand, appear to have distinct neural signatures, with differences in the parahippocampal structure and default mode connectivity that may be linked to maladaptive stress response and memory formation.

\section{Transdiagnostic association of cognitive function with neural signatures}

We identified impairments in different aspects of cognitive performance, including executive function in the four diagnostic groups, consistent with previous literature suggesting that executive dysfunction may be a transdiagnostic dimension of cognitive impairment $13,56,57$. Executive function impairments are not unique to mental disorders as physical problems, acute stress, or social isolation can also impact executive performance ${ }^{58}$.

While much is known about neural correlates of executive function ${ }^{59-63}$, lack of replication and small samples call for large-scale data ( $>2,000$ individuals) to uncover reproducible brain-wide associations with cognition ${ }^{64}$. In our large UKB sample, we found a specific functional connectivity profile between nodes of the frontoparietal, default mode and salience/attention networks and the cerebellum as underlying worse processing speed, spatial attention, fluid intelligence and paired associate learning. We showed that reduced withinnetwork and increased between-network connectivity of the frontoparietal and default mode networks predicted worse executive function and related cognitive impairments in MDD, anxiety and stress-related disorders. A similar pattern of brain-cognition relationships was found in the healthy control sample. These findings are consistent with previous multimodal meta-analyses showing that neural correlates of executive function in clinical populations encompass frontal, parietal, and cerebellar regions ${ }^{62}$ and previous large-scale analysis relating frontoparietal and default mode connectivity to worse verbal-numerical reasoning ${ }^{63}$ in UKB. Successful executive functioning relies on dynamic switching between frontoparietal and default mode networks initiated by cingulo-opercular attentional networks ${ }^{61,63}$. Self-referential processing in the default mode network is balanced with goal-oriented solving of complex problems (executive function) and influenced by salient events that can trigger the "switching" by attention networks.

Executive function itself is multidimensional, with processing speed, cognitive flexibility, fluid intelligence, working memory, planning, attention, and inhibitory control serving as interrelated sub-dimensions ${ }^{58}$. The second latent variable identified in our PLS analysis 
(PLS2) uncovered functional correlates of worse fluid intelligence and associative learning, which were impaired in the MDD and stressrelated disorder groups. In addition to connectivity pairs already identified as related to general cognitive function by the first PLS latent variable, PLS2 showed that higher connectivity of striatal areas with superior temporal gyri predicted better fluid intelligence and associative learning specifically. Identifying robust neural correlates of cognitive function using resting-state connectivity has been challenging due to the limitations of the sample sizes ${ }^{64}$ and the interindividual variability in brain function and its relation to cognitive performance ${ }^{65,66}$. Here we identify robust brain-cognition relationships that are replicable in a healthy population, furnishing evidence for existing theories of how functional organization of the brain supports is associated with cognitive dysfunction, including executive impairments.

We did not find the brain-cognition relationships identified across all diagnostic groups in each of the groups separately, however. Only MDD- and ANX- showed significant associations between cognitive performance on all four cognitive tests and functional connectivity captured by the first latent PLS variable. In these groups individually, different components of the default mode and frontoparietal networks were implicated. These findings suggest that the brain-cognition relationships uncovered here are not uniform across MDD and anxiety disorders. Instead, different aspects of frontoparietal and default mode interactions appear to underlie cognitive dysfunction in MDD and anxiety disorders.

\section{Limitations and Conclusions}

The cross-sectional nature of the data does not allow us to disambiguate whether the neural signatures are the consequence of, or a marker of vulnerability to, the respective disorder. Although diagnostic groups were defined by lifetime diagnosis, we found that approximately one-third of participants in our MDD or MDD with comorbid anxiety groups had some current depressive symptoms based

on the PHQ-2, ${ }^{35}$ ) at the time of cognitive and MRI assessment. Further, diagnoses were ascertained using electronic health records, that may show some heterogeneity 67.

While our analysis showed a similar signature of brain structure and function deviations across MDD and anxiety disorders, there are limitations to the degree of overlap between the disorders. No significant deviations in cortical thickness were seen in anxiety disorders, while the effects on cortical thickness seen in MDD and in the comorbid group were much larger and reached statistical significance. Interestingly, neuroimaging correlations accounted for less than $50 \%$ of variance in the disorder brain maps, suggesting that there are disorder-specific abnormalities in brain function and structure ${ }^{68}$.

In conclusion, we found a high degree of similarity in the neural signatures of MDD, anxiety disorders (alone and in comorbidity) that was distinct from stress-related disorders. Our findings are consistent with the diagnostic categorization of MDD and anxiety disorders as internalizing disorders (DSM 5). Stress-related disorders showed a very similar profile of executive dysfunction to MDD and anxiety disorders, yet their neural signatures showed less similarity, especially in the domain of cortical thickness. While the comorbidity across disorders is viewed as a therapeutic challenge, the identified neurobiological substrate of connectivity within and between default mode and frontoparietal networks that subserves cognitive dysfunction and could provide a promising target for specific interventions.

\section{Declarations}

\section{ACKNOWLEDGEMENTS}

\section{CONFLICTS \& DISCLOSURES}

PZ was funded by the Labatt Family Fellowship in Depression Biology and by the CIHR postdoctoral fellowship.

DF is supported by the generous contributions from the Michael and Sonja Koerner Foundation and the Krembil Family Foundation. DF is also supported in part by the CAMH Discovery Fund and CIHR.

BHM holds and receives support from the Labatt Family Chair in Biology of Depression in Late-Life Adults at the University of Toronto. He currently receives research support from Brain Canada, the Canadian Institutes of Health Research, the CAMH Foundation, the Patient-Centered Outcomes Research Institute (PCORI), the US National Institute of Health $(\mathrm{NIH})$, Capital Solution Design LLC (software used in a study founded by CAMH Foundation), and HAPPYneuron (software used in a study founded by Brain Canada). Within the past five years, he has also received research support from Eli Lilly (medications for a NIH-funded clinical trial) and Pfizer (medications for a NIH-funded clinical trial). He has been an unpaid consultant to Myriad Neuroscience. 
ANV currently receives funding from $\mathrm{ClHR}$, the $\mathrm{NIH}$, the National Sciences and Engineering Research Council (NSERC), the CAMH Foundation, and the University of Toronto.

AC has received research and consultancy fees from INCiPiT (Italian Network for Paediatric Trials), CARIPLO Foundation and Angelini Pharma. He is supported by the National Institute for Health Research (NIHR) Oxford Cognitive Health Clinical Research Facility, by an NIHR Research Professorship (grant RP-2017-08-ST2-006), by the NIHR Oxford and Thames Valley Applied Research Collaboration and by the NIHR Oxford Health Biomedical Research Centre (grant BRC-1215-20005). The views expressed are those of the authors and not necessarily those of the UK National Health Service, the NIHR, or the UK Department of Health.

\section{CONTRIBUTIONS}

Conception and design: All authors

Data analysis: PZ, MW

Data interpretation: All authors

Manuscript drafting and revision: All authors

Funding acquisition, project administration, and resources: ANV, DF

\section{References}

1. Hasin, D. S. et al. Epidemiology of adult DSM-5 major depressive disorder and its specifiers in the United States. JAMA Psychiatry 75, 336-346 (2018).

2. Beesdo, K., Pine, D. S., Lieb, R. \& Wittchen, H. U. Incidence and risk patterns of anxiety and depressive disorders and categorization of generalized anxiety disorder. Arch. Gen. Psychiatry 67, 47-57 (2010).

3. Lenze, E. J. et al. Generalized anxiety disorder in late life: Lifetime course and comorbidity with major depressive disorder. Am. J. Geriatr. Psychiatry 13, 77-80 (2005).

4. Kalin, N. H. The critical relationship between anxiety and depression. Am. J. Psychiatry 177, 365-367 (2020).

5. Anttila, V. et al. Analysis of shared heritability in common disorders of the brain. Science (80-. ). 360, (2018).

6. James, S. L. et al. Global, regional, and national incidence, prevalence, and years lived with disability for 354 Diseases and Injuries for 195 countries and territories, 1990-2017: A systematic analysis for the Global Burden of Disease Study 2017. Lancet 392, 1789-1858 (2018).

7. American Psychiatric Association. Diagnostic and Statistical Manual of Mental Disorders. APA Fifth Edit, (2013).

8. McTeague, L. M. et al. Identification of common neural circuit disruptions in cognitive control across psychiatric disorders. Am. J. Psychiatry 174, 676-685 (2017).

9. McTeague, L. M. et al. Identification of common neural circuit disruptions in emotional processing across psychiatric disorders. Am. J. Psychiatry 177, 411-421 (2020).

10. Goodkind, M. et al. Identification of a common neurobiological substrate for mental Illness. JAMA Psychiatry 72, 305-315 (2015).

11. Van Tol, M. J. et al. Regional brain volume in depression and anxiety disorders. Arch. Gen. Psychiatry 67, 1002-1011 (2010).

12. Radonjić, N. V. et al. Structural brain imaging studies offer clues about the effects of the shared genetic etiology among neuropsychiatric disorders. Mol. Psychiatry (2021). doi:10.1038/s41380-020-01002-z

13. Etkin, A. \& Cuthbert, B. Beyond the DSM: Development of a transdiagnostic psychiatric neuroscience course. Acad. Psychiatry 38, $145-150$ (2014). 
14. Janiri, D. et al. Shared Neural Phenotypes for Mood and Anxiety Disorders: A Meta-analysis of 226 Task-Related Functional Imaging Studies. JAMA Psychiatry 77, 172-179 (2020).

15. Sharp, P. B., Miller, G. A. \& Heller, W. Transdiagnostic dimensions of anxiety: Neural mechanisms, executive functions, and new directions. Int. J. Psychophysiol. 98, 365-377 (2015).

16. Feczko, E. et al. The Heterogeneity Problem: Approaches to Identify Psychiatric Subtypes. Trends Cogn. Sci. 23, 584-601 (2019).

17. Savulich, G. et al. Acute anxiety and autonomic arousal induced by $\mathrm{CO} 2$ inhalation impairs prefrontal executive functions in healthy humans. Transl. Psychiatry 9, (2019).

18. Shi, R., Sharpe, L. \& Abbott, M. A meta-analysis of the relationship between anxiety and attentional control. Clin. Psychol. Rev. 72, 101754 (2019).

19. Flaks, M. K. et al. Attentional and executive functions are differentially affected by post-traumatic stress disorder and trauma. J. Psychiatr. Res. 48, 32-39 (2014).

20. Aupperle, R. L., Melrose, A. J., Stein, M. B. \& Paulus, M. P. Executive function and PTSD: Disengaging from trauma. Neuropharmacology 62, 686-694 (2012).

21. Brewin, C. R. \& Holmes, E. A. Psychological theories of posttraumatic stress disorder. Clin. Psychol. Rev. 23, 339-376 (2003).

22. Durand, F., Isaac, C. \& Januel, D. Emotional memory in post-traumatic stress disorder: A systematic PRISMA review of controlled studies. Front. Psychol. 10, (2019).

23. Warren, S. L., Heller, W. \& Miller, G. A. The Structure of Executive Dysfunction in Depression and Anxiety. J. Affect. Disord. 279, 208-216 (2021).

24. Ashendorf, L. et al. Trail Making Test errors in normal aging, mild cognitive impairment, and dementia. Arch. Clin. Neuropsychol. 23, $129-137$ (2008).

25. Rosano, C. et al. Digit symbol substitution test and future clinical and subclinical disorders of cognition, mobility and mood in older adults. Age Ageing 45, 687-694 (2016).

26. Matsuda, O. \& Saito, M. Crystallized and fluid intelligence in elderly patients with mild dementia of the Alzheimer type. Int. Psychogeriatrics 10, 147-154 (1998).

27. Pike, K. E., Rowe, C. C., Moss, S. A. \& Savage, G. Memory Profiling With Paired Associate Learning in Alzheimer's Disease, Mild Cognitive Impairment, and Healthy Aging. Neuropsychology 22, 718-728 (2008).

28. Kaiser, R. H., Andrews-Hanna, J. R., Wager, T. D. \& Pizzagalli, D. A. Large-scale network dysfunction in Major Depressive Disorder: Meta-analysis of resting-state functional connectivity. JAMA Psychiatry 72, 603-611 (2015).

29. Xu, J. et al. Anxious brain networks: A coordinate-based activation likelihood estimation meta-analysis of resting-state functional connectivity studies in anxiety. Neurosci. Biobehav. Rev. 96, 21-30 (2019).

30. Modi, S., Kumar, M., Kumar, P. \& Khushu, S. Aberrant functional connectivity of resting state networks associated with trait anxiety. Psychiatry Res. - Neuroimaging 234, 25-34 (2015).

31. Schmaal, L. et al. Cortical abnormalities in adults and adolescents with major depression based on brain scans from 20 cohorts worldwide in the ENIGMA Major Depressive Disorder Working Group. Mol. Psychiatry 22, 900-909 (2017).

32. Gray, J. P., Müller, V. I., Eickhoff, S. B. \& Fox, P. T. Multimodal Abnormalities of Brain Structure and Function in Major Depressive Disorder: A Meta-Analysis of Neuroimaging Studies. Am. J. Psychiatry appi.ajp.2019.1 (2020). doi:10.1176/appi.ajp.2019.19050560

33. Pink, A. et al. Cortical thickness and anxiety symptoms among cognitively normal elderly persons: The mayo clinic study of aging. J. Neuropsychiatry Clin. Neurosci. 29, 60-66 (2017). 
34. Jankord, R. \& Herman, J. P. LIMBIC REGULATION OF HYPOTHALAMO-PITUITARY- ADRENOCORTICAL FUNCTION DURING ACUTE AND CHRONIC STRESS. Ann N Y Acad Sci. 1148, 64-73 (2008).

35. Levis, B. et al. Accuracy of the PHQ-2 Alone and in Combination with the PHQ-9 for Screening to Detect Major Depression: Systematic Review and Meta-analysis. JAMA - J. Am. Med. Assoc. 323, 2290-2300 (2020).

36. Miller, K. L. et al. Multimodal population brain imaging in the UK Biobank prospective epidemiological study. Nat. Neurosci. 19, $1523-1536$ (2016).

37. Dale, A. M., Fischl, B. \& Sereno, M. I. Cortical surface-based analysis. I. Segmentation and Surface Reconstruction. Neuroimage 9, 195-207 (1999).

38. Desikan, R. S. et al. An automated labeling system for subdividing the human cerebral cortex on MRI scans into gyral based regions of interest. Neuroimage 31, 968-980 (2006).

39. Alfaro-Almagro, F. et al. Image processing and Quality Control for the first 10,000 brain imaging datasets from UK Biobank. Neuroimage 166, 400-424 (2018).

40. Yeo, B. T. et al. The organization of the human cerebral cortex estimated by intrinsic functional connectivity. J. Neurophysiol. 106, $1125-1165$ (2011).

41. Glasser, M. F. et al. A multi-modal parcellation of human cerebral cortex. Nature 536, 171-178 (2016).

42. Salthouse, T. A. What cognitive abilities are involved in trail-making performance? Intelligence 39, 222-232 (2011).

43. Norbert M. Seel. Encyclopedia of the Sciences of Learning. Encyclopedia of the Sciences of Learning (2012). doi:10.1007/978-14419-1428-6

44. Jaeger, J. Digit symbol substitution test. J. Clin. Psychopharmacol. 38, 513-519 (2018).

45. Wainberg, M. et al. Symptom dimensions of major depression in a large community-based cohort. (2021).

46. Meier, S. M. et al. Genetic Variants Associated with Anxiety and Stress-Related Disorders: A Genome-Wide Association Study and Mouse-Model Study. JAMA Psychiatry 76, 924-932 (2019).

47. Nievergelt, C. M. et al. International meta-analysis of PTSD genome-wide association studies identifies sex- and ancestry-specific genetic risk loci. Nat. Commun. 10, 1-16 (2019).

48. Anderson, K. M. et al. Convergent molecular, cellular, and cortical neuroimaging signatures of major depressive disorder. Proc. Natl. Acad. Sci. 117, 202008004 (2020).

49. Morgan, S. E. et al. Cortical patterning of abnormal morphometric similarity in psychosis is associated with brain expression of schizophrenia-related genes. Proc. Natl. Acad. Sci. 116, 9604-9609 (2019).

50. Spijker, J., Muntingh, A. \& Batelaan, N. Advice for Clinicians onHow to Treat Comorbid Anxiety and Depression. Depress. Anxiety 31, 934-940 (2014).

51. Saade, Y. M. et al. Comorbid anxiety in late-life depression: Relationship with remission and suicidal ideation on venlafaxine treatment. Depress. Anxiety 36, 1125-1134 (2019).

52. Shin, L. M. \& Liberzon, I. The neurocircuitry of fear, stress, and anxiety disorders. Neuropsychopharmacology 35, 169-191 (2010).

53. Morey, R. A. et al. Amygdala volume changes in posttraumatic stress disorder in a large case-controlled veterans group. Arch. Gen. Psychiatry 69, 1169-1178 (2012).

54. Furman, D. J., Hamilton, J. P. \& Gotlib, I. H. Frontostriatal functional connectivity in major depressive disorder. Biol. Mood Anxiety Disord. 1, (2011). 
55. Howard, D. M. et al. Genome-wide association study of depression phenotypes in UK Biobank identifies variants in excitatory synaptic pathways. Nat. Commun. 9, 1-10 (2018).

56. Ittenger, C. \& Etkin, A. Are there biological commonalities amongdifferent psychiatric disorders? in Psychiatry (3rd edition) 245256 (2008).

57. Zelazo, P. D. Executive Function and Psychopathology: A Neurodevelopmental Perspective. Annu. Rev. Clin. Psychol. 16, 431-454 (2020).

58. Diamond, A. Executive Function. Annu. Rev. Psychol. 64, 136-168 (2013).

59. Whelan, R. et al. Adolescent impulsivity phenotypes characterized by distinct brain networks. Nat. Neurosci. 15, $920-925$ (2012).

60. Menon, V. Large-scale brain networks and psychopathology: a unifying triple network model. Trends Cogn. Sci. $x x$ 1-24 (2011). doi:10.1016/j.tics.2011.08.003

61. Menon, V. Large-Scale Brain Networks in Cognition: Emerging Principles. Neogene Quat. Dinoflag. cysts acritarchs 121-132 (2010).

62. Nowrangi, M. A., Lyketsos, C., Rao, V. \& Munro, C. A. Systematic review of neuroimaging correlates of executive functioning: Converging evidence from different clinical populations. J. Neuropsychiatry Clin. Neurosci. 26, 114-125 (2014).

63. Shen, X. et al. Resting-State Connectivity and Its Association With Cognitive Performance, Educational Attainment, and Household Income in the UK Biobank. Biol. Psychiatry Cogn. Neurosci. Neuroimaging 3, 878-886 (2018).

64. Marek, S. et al. Towards Reproducible Brain-Wide Association Studies Affiliations. bioRxiv 15-18 (2020).

65. Seghier, M. L. \& Price, C. J. Interpreting and Utilising Intersubject Variability in Brain Function. Trends Cogn. Sci. 22, 517-530 (2018).

66. Li, R. et al. Linking inter-individual variability in functional brain connectivity to cognitive ability in elderly individuals. Front. Aging Neurosci. 9, 1-13 (2017).

67. Vaci, N. et al. Natural language processing for structuring clinical text data on depression using UK-CRIS. Evid. Based. Ment. Health 23, 21-26 (2020).

68. Oathes, D. J., Patenaude, B., Schatzberg, A. F. \& Etkin, A. Neurobiological signatures of anxiety and depression in resting-state functional magnetic resonance imaging. Biol. Psychiatry 77, 385-393 (2015).

\section{Figures}



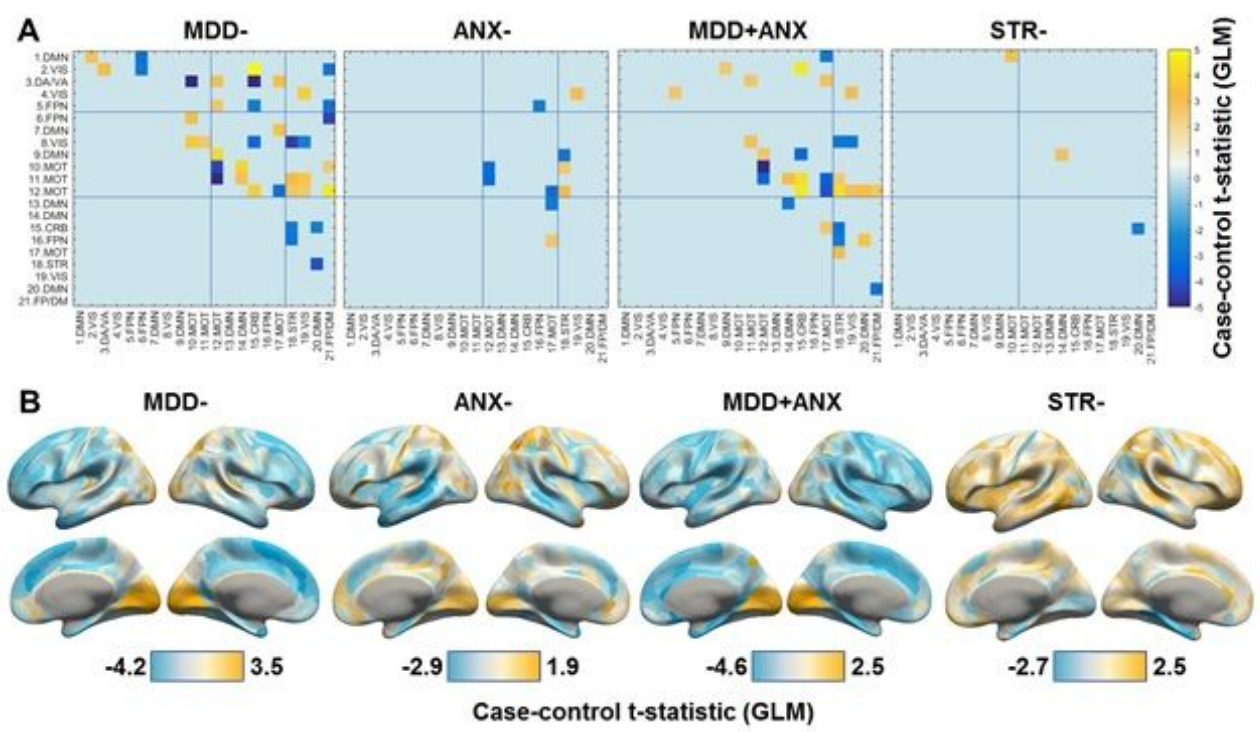

Figure 1

Case-control differences in functional connectivity (A) and cortical thickness (B), not covarying for the effects of polygenic risk. Casecontrol t-statistics of $t 27,120=5$ correspond to an effect size $d=0.061$ for resting state connectivity, and t30,419=5 to $d=0.057$ for cortical thickness. Lower half of the correlation matrix is left blank.

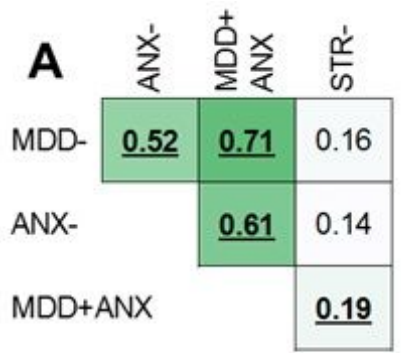

Resting-state connectivity

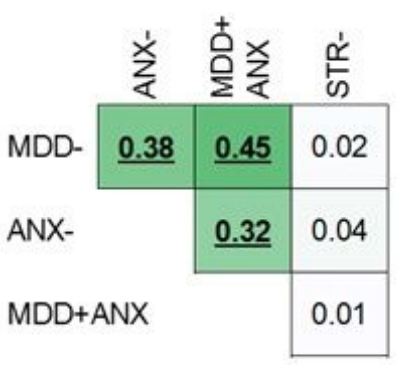

Cortical thickness
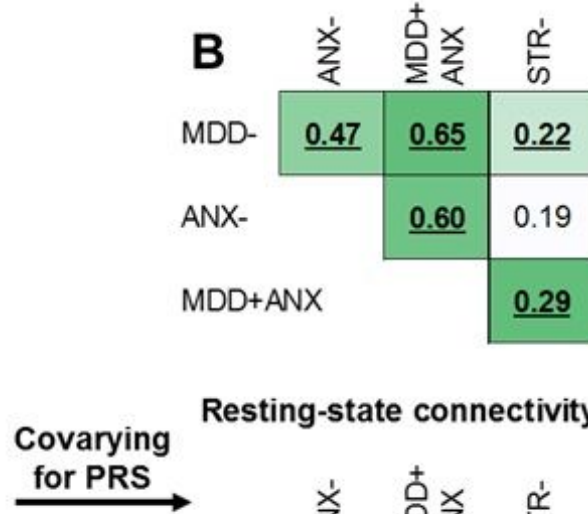

0.7

$\simeq$

Resting-state connectivity

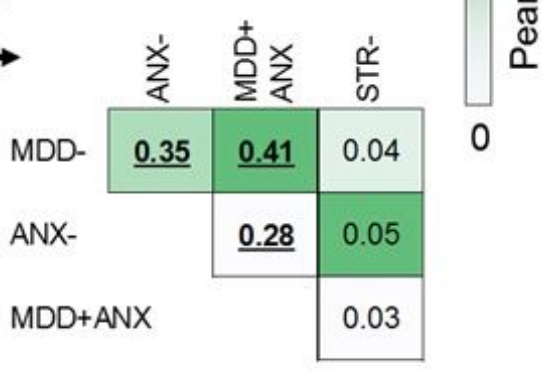

Cortical thickness

\section{Figure 2}

Disorder similarity matrices for resting-state connectivity and cortical thickness (A). The values represent Pearson's correlations of casecontrol or PRS statistics from the 210 resting-state connectivities and 360 regional cortical thicknesses. The disorder similarity matrices were slightly smaller when the three PRS for MDD, anxiety disorders, and post-traumatic stress disorder were included as covariates (B). Significant correlations at PPERM $<0.01$ are shown in bold and underlined. ANX - anxiety, MDD - major depressive disorder; STR stress-related disorders. 

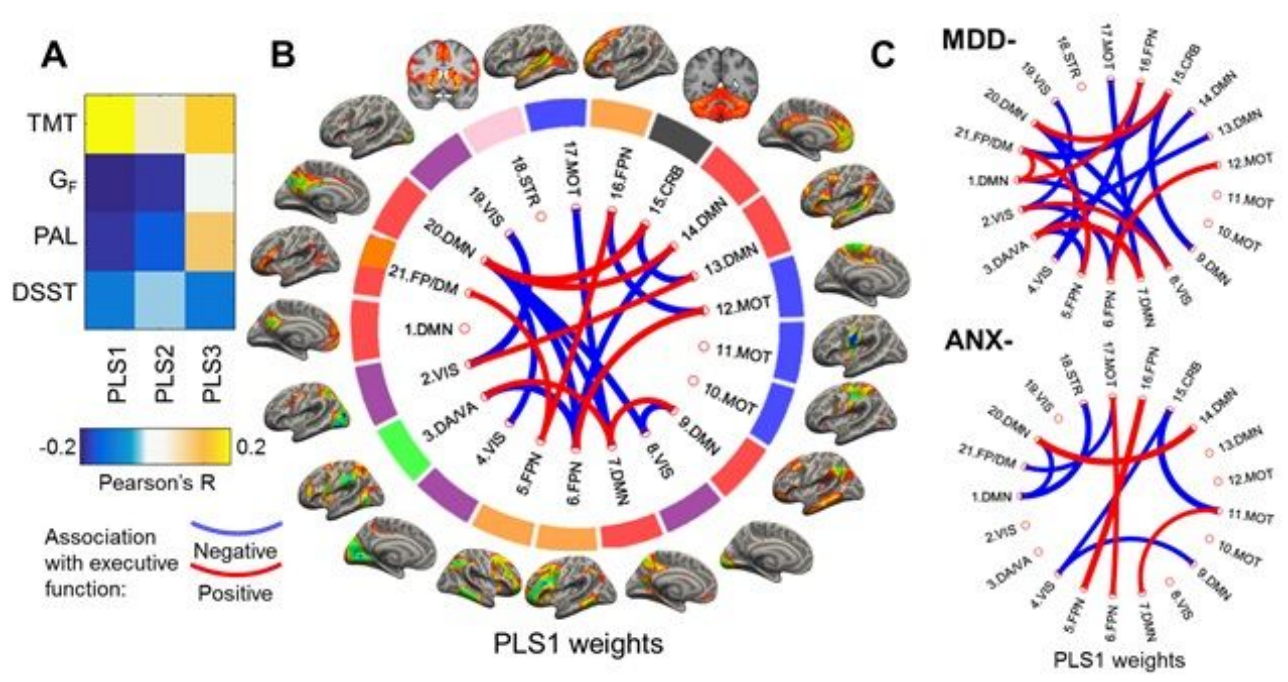

Figure 3

Dimensional relationships between functional connectivity and cognitive function from a partial least squares (PLS) regression in participants with major depression, anxiety, or stress-related disorders. PLS latent variable 1 (PLS1) accounted for the largest amount of variance in cognitive tests. Higher loadings on PLS1 were associated with worse cognitive performance on all four tests (A), characterized by longer times to complete the Trailmaking path (TMT), lower number of correct reasoning questions in the fluid intelligence test (Gf), lower number of word-pairs recalled on the paired associate learning (PAL) test and lower number of digits being filled in in the digit-symbol substitution test (DSST). Thresholded PLS1 weights ( $Z>3$ and $Z<-3$ ) implicated pairwise connectivities between independent components corresponding to the default mode (DMN), frontoparietal (FPN) and dorsal/ventral attention (DA/VA) networks (B). Blue connections between network components suggest that higher connectivity of those components was associated with worse cognitive performance. Red connections between network components suggest that higher connectivity of those components predicted better cognitive performance. (C) Repeating the PLS regressions in each case group separately revealed that the braincognition relationships were driven by MDD- and ANX- groups, with no significant relationships found in MDD+ANX or STR-. Other network labels include MOT - motor; CRB - cerebellum; VIS - visual; STR - striatum.

\section{Supplementary Files}

This is a list of supplementary files associated with this preprint. Click to download.

- SupplementaryInformation.docx 